\title{
Molecular gas conditions in NGC 4945 and the Circinus galaxy ${ }^{\star}$
}

\author{
S. J. Curran ${ }^{1,2}$, L. E. B. Johansson ${ }^{1}$, P. Bergman ${ }^{1}$, A. Heikkilä ${ }^{1,3}$, and S. Aalto ${ }^{1}$ \\ 1 Onsala Space Observatory, Chalmers University of Technology, 43992 Onsala, Sweden \\ 2 European Southern Observatory, Casilla 19001, Santiago 19, Chile \\ 3 Observatory, PO Box 14, 00014 University of Helsinki, Finland
}

Received 11 July 2000 / Accepted 5 December 2000

\begin{abstract}
We present results of a multi-transition study of the dense molecular gas in the central part of the hybrid star-burst/Seyfert galaxies NGC 4945 and the Circinus galaxy. From the results of radiative transfer calculations, we estimate in NGC $4945 n_{\mathrm{H}_{2}}=310^{3}-10^{4} \mathrm{~cm}^{-3}$ and $T_{\text {kin }} \approx 100 \mathrm{~K}$ and in Circinus $n_{\mathrm{H}_{2}}=$ $210^{3}-10^{5} \mathrm{~cm}^{-3}$ and $T_{\text {kin }} \approx 50-80 \mathrm{~K}$ for the molecular hydrogen density and kinetic temperature, respectively. As well as density/temperature tracing molecules, we have observed $\mathrm{C}^{17} \mathrm{O}$ and $\mathrm{C}^{18} \mathrm{O}$ in each galaxy and the value of $\mathrm{C}^{18} \mathrm{O} / \mathrm{C}^{17} \mathrm{O} \approx 6$ for the isotopic column density ratio suggests that both have relatively high populations of massive stars. Finally, although star formation is present, the radiative transfer results combined with the high $\mathrm{HCN} / \mathrm{CO}$ and (possibly) HCN/FIR, radio/FIR ratios may suggest that, in comparison with Circinus, a higher proportion of the dense gas emission in NGC 4945 may be located in the hypothesised central nuclear disk as opposed to dense star forming cloud cores. Contrary to the literature, which assumes that all of the far-infrared emission arises from star formation, our results suggest that in NGC 4945 some of this emission could arise from an additional source, and so we believe that a revision of the star formation rate estimates may be required for these two galaxies.
\end{abstract}

Key words. galaxies: abundances - galaxies: individual: NGC 4945, Circinus - galaxies: nuclei - galaxies: Seyfert - galaxies: star-burst

\section{Introduction}

Seyfert galaxies account for $\sim 10 \%$ of all galaxies (Maiolino \& Rieke 1995; Ho et al. 1997) and along with LINERs ${ }^{1}$ and radio quiet quasars constitute one of the three main classes of active galactic nuclei (AGNs); the other classes being the more luminous FRI radio galaxies/BL Lac objects and the most luminous FRII radio galaxies/quasars. Although sometimes associated with enhanced star-burst activity (e.g. Whittle 1992; Gu et al. 1997; Roy et al. 1998; Gu et al. 1999), a Seyfert nucleus exhibits the telltale signs of an AGN via a non-stellar nuclear source (Rigopoulou et al. 1997) and unlike star-burst galaxies, the nuclei are often associated with radio or optical jets (Hummel et al. 1983; de Grijp et al. 1985; Cecil et al. 1992 $)^{2}$. Recently, Curran et al. (2000) have shown, from

Send offprint requests to: S. J. Curran,

e-mail: scurran@eso.org

* Based on results collected at the European Southern Observatory, La Silla, Chile.

${ }^{1}$ Low Ionisation Nuclear Emission-line Regions.

2 Due to ionised gas (Storchi-Bergmann et al. 1995) ejected from a compact nucleus (Whittle et al. 1988; Pedlar et al. 1989; Christopoulou et al. 1997; Radovich et al. 1998). a sample of 20 Seyfert galaxies, that the HCN/CO luminosity ratio is similar to that of ultra-luminous infrared galaxies (ULIRGs), while being significantly higher than that of normal spiral galaxies; $\frac{L_{\mathrm{HCN}}}{L_{\mathrm{CO}}} \approx 1 / 6$ for the distant sources $\left(L_{\mathrm{FIR}} \approx 3010^{10} L_{\odot}\right)$ and $\frac{L_{\mathrm{HCN}}}{L_{\mathrm{CO}}} \approx 1 / 17$ for the near-by sources $\left(L_{\mathrm{FIR}} \approx 210^{10} L_{\odot}\right)$ (Curran et al. 2000), cf. $\frac{L_{\mathrm{HCN}}}{L_{\mathrm{CO}}} \approx 1 / 80$ for normal spirals (Solomon et al. 1992). The fraction of the HCN brightness which arises from the AGN (via the water maser disks ${ }^{3}$ ) or from star forming cores is still, however, uncertain (e.g. Kohno et al. 1999).

NGC 4945 has its visual appearance dominated by strong and patchy extinction in the disk, which hides any distinct visible nucleus, although a relatively strong and compact $\left(30^{\prime \prime}\right)$ non-thermal radio source, which is coincident with the peak of red and infra-red emission (Moorwood \& Glass 1984), is visible in the central region. Also, wide absorption lines $\left(\mathrm{OH}, \mathrm{CH}, \mathrm{H}_{2} \mathrm{CO}\right)$ are found towards the radio-continuum nucleus (Whiteoak \& Gardner 1975; Whiteoak \& Gardner 1985; Ables et al. 1987; Whiteoak \& Wilson 1990). Towards this position

3 These pc-scale molecular disks are believed to be associated with the central body responsible for the obscuration of the nuclei in Seyfert galaxies (Antonucci 1993). See Curran (2000) (http://nedwww.ipac.caltech.edu/level5/Curran/ frames.html) for a review. 
a very powerful $\mathrm{H}_{2} \mathrm{O}$ maser has been detected (dos Santos \& Lépine 1979; Batchelor et al. 1982; Greenhill et al. 1997). The nucleus exhibits star-burst as well as Seyfert characteristics and has properties similar to that of Circinus although for some species the molecular line characteristics differ.

The Circinus galaxy also has $\mathrm{H}_{2} \mathrm{O}$ maser activity (Gardner \& Whiteoak 1982; Greenhill et al. 1995; Greenhill et al. 1997; Greenhill 2000) the high luminosity of which suggests the presence of an active galactic nucleus (AGN). Again, this galaxy is host to nuclear starburst activity (Harnett et al. 1990; Moorwood et al. 1996a) and the presence of visible and near infra-red coronal lines (Oliva et al. 1994), an X-ray reflection dominated spectrum (Matt et al. 1996) and broad polarised $\mathrm{H} \alpha$ (Oliva et al. 1998) suggest the presence of a hidden Seyfert nucleus.

These almost edge-on near-by Southern galaxies (Freeman et al. 1977; de Vaucouleurs et al. 1981; Ables et al. 1987) are similar, in that:

1. Both possess molecular rings with very similar properties, which are associated with the star-burst activity (Bergman et al. 1992; Dahlem et al. 1993; Marconi et al. 1994; Curran et al. 1998);

2. Both galaxies have a dynamical mass of $310^{9} M_{\odot}$ within the central 600 pc (Mauersberger et al. 1996; Curran et al. 1998);

3. They also share similar X-ray properties (see Spoon et al. 2000).

Because of the large variety of molecules (at least in NGC 4945, Whiteoak et al. 1990; Henkel et al. 1994) and the proximity of the galaxies, extraordinarily strong emission lines from a number of molecules may be observed. Thus a relatively detailed observational study of the chemical and physical properties of the molecular gas in the central parts of these galaxies is possible, even with the sensitivity and the angular resolution of the present day single dish millimetre-wave telescopes. We have observed spectral line emission from a number of molecules (some in several transitions) and extend the previous studies of Whiteoak et al. (1990); Bergman et al. (1992); Henkel et al. (1994) and present our results in this paper.

\section{Observations and results}

The observations were carried out in June 1989, February 1993, June 1995, June 1998, December 1998, December 1999 and April 2000 with the Swedish-ESO Submillimetre Telescope (SEST) ${ }^{4}$ at La Silla, Chile. The receiver system consisted of a $3 \mathrm{~mm}$ Schottky mixer and cryogenic $1.3 \mathrm{~mm}, 2 \mathrm{~mm}$ and $3 \mathrm{~mm}$ SIS mixers. The mixers were tuned to single sideband mode (resulting system

\footnotetext{
${ }^{4}$ The Swedish-ESO Sub-millimetre Telescope is operated jointly by ESO and the Swedish National Facility for Radio Astronomy, Onsala Space Observatory at Chalmers University of Technology.
}

Table 1. The integrated line intensities observed towards the centre of NGC 4945; the detections are shown in Fig. 1. Here and in Table 2 , the uncertainties are due to $1 \sigma$ rms noise fluctuations and the $\approx \pm 5 \%$ accuracy of $\eta_{\mathrm{mb}}$. The upper limits where obtained by assuming the same velocity range as for the $\mathrm{CO}$ (and other molecules), i.e. $\approx 300 \mathrm{~km} \mathrm{~s}^{-1}$ to $\approx 800 \mathrm{~km} \mathrm{~s}^{-1}$. The $\mathrm{H}_{2} \mathrm{CO} J_{\mathrm{K}_{\mathrm{a}}, \mathrm{K}_{\mathrm{c}}}=2_{0,2} \rightarrow 1_{0,1}$ line is a blend with $\mathrm{HC}_{3} \mathrm{~N}$ $J=16 \rightarrow 15$

\begin{tabular}{|c|c|c|}
\hline Molecule & Transition & $I_{\mathrm{mb}}\left[\mathrm{K} \mathrm{km} \mathrm{s}^{-1}\right]$ \\
\hline \multirow[t]{3}{*}{${ }^{12} \mathrm{CO}$} & $1 \rightarrow 0$ & $510 \pm 30$ \\
\hline & $2 \rightarrow 1$ & $740 \pm 40$ \\
\hline & $3 \rightarrow 2$ & $760 \pm 50$ \\
\hline \multirow[t]{2}{*}{${ }^{13} \mathrm{CO}$} & $1 \rightarrow 0$ & $30 \pm 2$ \\
\hline & $2 \rightarrow 1$ & $86 \pm 5$ \\
\hline \multirow[t]{2}{*}{$\mathrm{C}^{18} \mathrm{O}$} & $1 \rightarrow 0$ & $8.4 \pm 0.8$ \\
\hline & $2 \rightarrow 1$ & $29 \pm 2$ \\
\hline \multirow[t]{2}{*}{$\mathrm{C}^{17} \mathrm{O}$} & $1 \rightarrow 0$ & $1.8 \pm 0.4$ \\
\hline & $2 \rightarrow 1$ & $5 \pm 1$ \\
\hline \multirow[t]{2}{*}{ CS } & $2 \rightarrow 1$ & $9.3 \pm 0.8$ \\
\hline & $3 \rightarrow 2$ & $11 \pm 1$ \\
\hline${ }^{13} \mathrm{CS}$ & $2 \rightarrow 1$ & $<2$ \\
\hline \multirow[t]{2}{*}{$\mathrm{SO}$} & $3_{2} \rightarrow 2_{1}$ & $1.2 \pm 0.3$ \\
\hline & $4_{3} \rightarrow 3_{2}$ & $1.4 \pm 0.2$ \\
\hline \multirow[t]{2}{*}{$\mathrm{HCN}$} & $1 \rightarrow 0$ & $24 \pm 2$ \\
\hline & $3 \rightarrow 2$ & $45 \pm 4$ \\
\hline $\mathrm{H}^{13} \mathrm{CN}$ & $1 \rightarrow 0$ & $1.1 \pm 0.4$ \\
\hline \multirow[t]{2}{*}{$\mathrm{HCO}^{+}$} & $1 \rightarrow 0$ & $21 \pm 1$ \\
\hline & $3 \rightarrow 2$ & $37 \pm 3$ \\
\hline \multirow[t]{2}{*}{$\mathrm{H}^{13} \mathrm{CO}^{+}$} & $1 \rightarrow 0$ & $2.3 \pm 0.5$ \\
\hline & $3 \rightarrow 2$ & $<4$ \\
\hline \multirow[t]{6}{*}{$\mathrm{H}_{2} \mathrm{CO}$} & $2_{1,2} \rightarrow 1_{1,1}$ & $6.5 \pm 0.7$ \\
\hline & $2_{0,2} \rightarrow 1_{0,1}$ & $5.4 \pm 0.5$ \\
\hline & $2_{1,1} \rightarrow 1_{1,0}$ & $5.9 \pm 0.6$ \\
\hline & $3_{0,3} \rightarrow 2_{0,2}$ & $<3$ \\
\hline & $3_{2,2} \rightarrow 2_{2,1}$ & $<3$ \\
\hline & $3_{1,2} \rightarrow 2_{1,1}$ & $<3$ \\
\hline $\mathrm{CH}_{3} \mathrm{C}_{2} \mathrm{H}$ & $6 \rightarrow 5$ & $<2$ \\
\hline
\end{tabular}

temperatures, corrected for the atmosphere, were typically $\left.T_{\mathrm{A}}^{*} \approx 200-550 \mathrm{~K}\right)$, and connected to low resolution acousto-optical spectrometers (1440 channels, total bandwidth of $1 \mathrm{GHz}$ ). Dual beam-switching, with a beamthrow of $\approx 12^{\prime}$ in azimuth, was used as observing mode. The intensity calibration was done with the chopper-wheel method. Pointing and focus checks were made towards stellar $\mathrm{SiO}$ masers as well as the continuum source in Centaurus A. The pointing offsets were typically $3^{\prime \prime} \mathrm{rms}$ in each coordinate. Calibration uncertainties are estimated to be $\pm 10,15$ and $20 \%$ in the 3,2 and $1.3 \mathrm{~mm}$ bands, respectively.

\subsection{Observational results}

Figures 1 and 2 show the observed spectra. The observational results are summarised in Tables 1 and 2, where 
Table 2. The integrated line intensities observed towards the centre of Circinus; the detections are shown in Fig. 2. The CO data are taken from Curran et al. (1998). As in the case of NGC 4945 (Table 1), the integrated intensities were estimated by assuming the same velocity range as for the $\mathrm{CO}$ i.e. $\approx 200 \mathrm{~km} \mathrm{~s}^{-1}$ to $\approx 650 \mathrm{~km} \mathrm{~s}^{-1}$

\begin{tabular}{lcr}
\hline Molecule & Transition & $I_{\mathrm{mb}}\left[\mathrm{K} \mathrm{km} \mathrm{s}^{-1}\right]$ \\
\hline${ }^{12} \mathrm{CO}$ & $1 \rightarrow 0$ & $180 \pm 10$ \\
& $2 \rightarrow 1$ & $220 \pm 20$ \\
& $3 \rightarrow 2$ & $230 \pm 20$ \\
${ }^{13} \mathrm{CO}$ & $1 \rightarrow 0$ & $12 \pm 1$ \\
& $2 \rightarrow 1$ & $24 \pm 4$ \\
$\mathrm{C}^{18} \mathrm{O}$ & $1 \rightarrow 0$ & $4.3 \pm 0.4$ \\
$\mathrm{C}^{17} \mathrm{O}$ & $1 \rightarrow 0$ & $0.9 \pm 0.1$ \\
$\mathrm{CS}$ & $2 \rightarrow 1$ & $3.2 \pm 0.3$ \\
& $3 \rightarrow 2$ & $3.0 \pm 0.3$ \\
& $5 \rightarrow 4$ & $1.1 \pm 0.3$ \\
$\mathrm{SO}$ & $32 \rightarrow 21$ & $0.8 \pm 0.2$ \\
$\mathrm{HCN}$ & $1 \rightarrow 0$ & $5.2 \pm 0.8$ \\
$\mathrm{HNC}$ & $1 \rightarrow 0$ & $3 \pm 1$ \\
$\mathrm{HCO}{ }^{+}$ & $1 \rightarrow 0$ & $7 \pm 1$ \\
$\mathrm{H}_{2} \mathrm{CO}$ & $21,2 \rightarrow 1_{1,1}$ & $0.8 \pm 0.2$ \\
\hline
\end{tabular}

main-beam integrated intensities are given ${ }^{5}$. The total uncertainty in the observed intensities is obtained by adding quadratically the contribution of noise in the spectra to the calibration and pointing uncertainties.

In NGC 4945 most of the molecules have stronger emission in the blue-shifted than in the red-shifted part of the line. An exception to this is CS, whose emission is more evenly distributed over the full spectral profile (this has previously been noted by Henkel et al. 1990). This asymmetry ${ }^{6}$ in the spectra may indicate that the chemistries differ in the two regions or that the excitation is varying, e.g. due to differences in the gas density/temperature, electron density or the background infrared radiation. Henkel et al. (1994) have suggested that the differing emission strengths may be due to differences in the exposure to the far-infrared (FIR) radiation. Yet another possible explanation is self-absorption, i.e. some locations suffering a greater degree of saturation (Henkel et al. 1990), perhaps due to a temperature gradient across the molecular ring where the inner edge is expected to be hotter than the outer.

Unlike those of NGC 4945 the profiles in Circinus are fairly symmetric. Again checking against previous observations, the lines are similar to those which have been previously observed i.e. ${ }^{12} \mathrm{CO} 1 \rightarrow 0$ (Johansson et al. 1991; Israel 1992; Elmouttie et al. 1997; Elmouttie et al. 1998),

\footnotetext{
5 The main beam brightness temperature is defined by $T_{\mathrm{mb}}=$ $T_{\mathrm{A}}^{*} / \eta_{\mathrm{mb}}$, where $T_{\mathrm{A}}^{*}$ is the Rayleigh-Jeans antenna temperature above the atmosphere and $\eta_{\mathrm{mb}}$ is the measured main-beam efficiency of the telescope. The velocity integrated intensity is given by $I_{\mathrm{mb}}=\int T_{\mathrm{mb}} \mathrm{d} v$.

${ }^{6}$ Also seen by Henkel et al. 1990; Henkel et al. (1994).
}

Table 3. The beam widths, $\Theta_{\mathrm{mb}}$, at SEST and source sizes, $\Theta_{\text {source }}$, of the various CO transitions. In the case of NGC 4945 the $1 \rightarrow 0$ value is from Dahlem et al. (1993); Mauersberger et al. (1996), the $2 \rightarrow 1$ value from Dahlem et al. (1993); Mauersberger et al. (1996); Curran (2000) and the $3 \rightarrow 2$ value from Mauersberger et al. (1996); Curran (2000). For Circinus all the values are obtained from the maps of Curran et al. (1998). Applying the FWHMs listed to the relation $I=I_{\mathrm{mb}} / \eta_{\mathrm{bf}}\left(\right.$ where $\left.\eta_{\mathrm{bf}}=\Theta_{\text {source }}^{2} /\left(\Theta_{\mathrm{mb}}^{2}+\Theta_{\text {source }}^{2}\right)\right)$, in order to correct for the Gaussian beam-filling factor, we obtain the corrected intensity ratios listed in Table 4

\begin{tabular}{lccc}
\hline & CO $1 \rightarrow 0$ & CO $2 \rightarrow 1$ & CO $3 \rightarrow 2$ \\
\hline SEST beam & $45^{\prime \prime}$ & $22^{\prime \prime}$ & $15^{\prime \prime}$ \\
NGC 4945 & $29^{\prime \prime}$ & $20^{\prime \prime}$ & $15^{\prime \prime}$ \\
Circinus & $42^{\prime \prime}$ & $31^{\prime \prime}$ & $21^{\prime \prime}$ \\
\hline
\end{tabular}

${ }^{13} \mathrm{CO} 1 \rightarrow 0,{ }^{12} \mathrm{CO} 2 \rightarrow 1,{ }^{13} \mathrm{CO} 2 \rightarrow 1$ (Johansson et al. 1991) and $\mathrm{HCO}^{+}, \mathrm{HCN}$ and $\mathrm{HNC}$, in the $1 \rightarrow 0$ transition (Israel 1992).

\subsection{Excitation and radiative transfer analysis}

In the analysis we use velocity integrated line intensities, $I=\int T \mathrm{~d} v$, which have been corrected for beamdilution due to finite source and beam sizes. This is carried out by assuming that the source distribution on the sky is Gaussian and applying the full-width halfmaximum $(\mathrm{FWHM})$ diameter $\left(\Theta_{\text {source }}\right)$ of the corresponding ${ }^{12} \mathrm{CO}$ transition to the various $\mathrm{CO}$ isotopomers. The other molecules have transitions of higher excitation requirements and in those cases the $\mathrm{CO} 2 \rightarrow 1$ value of $\Theta_{\text {source }}$ is applied (Table 3). The resulting corrected intensity ratios are shown in Table 4.

For both galaxies we find a ${ }^{12} \mathrm{CO} 2 \rightarrow 1 / 1 \rightarrow 0$ intensity ratio of $\approx 1$ which is typical for star-burst/Seyfert galaxies (Aalto et al. 1991; Dahlem et al. 1993) ${ }^{7}$. Also, the $\mathrm{CO} 1 \rightarrow 0 / \mathrm{HCN} 1 \rightarrow 0$ intensity ratios are similar to those found in (other) Seyfert galaxies; with NGC 4945 showing the same ratio as for the more distant Seyferts and Circinus showing a similar ratio to the near-by sample (see Sect. 1). In NGC 4945 our ${ }^{12} \mathrm{CO}$ and ${ }^{13} \mathrm{CO} 2 \rightarrow 1 / 1 \rightarrow 0$ ratio agrees with that previously determined (e.g. Dahlem et al. 1993; Henkel et al. 1994).

In order to estimate the prevailing physical conditions and column densities in the molecular gas, we have performed radiative transfer calculations. In order to complement this, in temperature and column density estimates we have also applied local thermodynamic equilibrium (LTE) calculations. The presented column density estimates are peak values in the sense that the intensities have been corrected for finite source and beam size. However, small-scale (i.e. structures smaller than the

\footnotetext{
7 Papadopoulos \& Seaquist (1998) find ratios somewhat lower than this, although Curran et al. (2000) and Curran (2000) suggest that these may be due to pointing errors.
} 

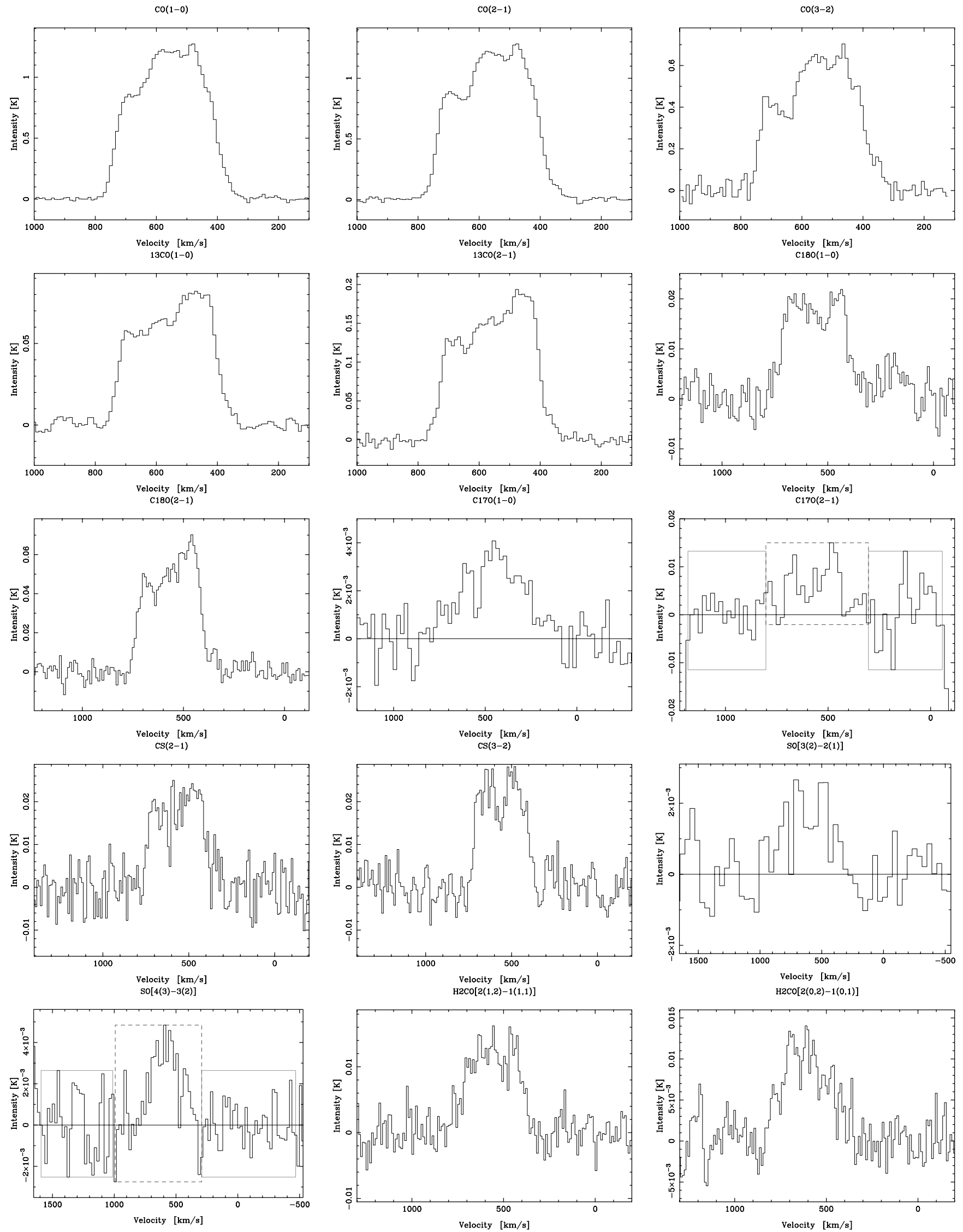

Fig. 1. The observed spectra in NGC 4945. Apart from $\mathrm{H}_{2} \mathrm{CO} 2_{1,1} \rightarrow 1_{1,0}$, which required the removal of a higher order baseline, a constant or first order baseline has been subtracted from each spectra. The spectra have been smoothed to a channel width corresponding to $10 \mathrm{~km} \mathrm{~s}^{-1}$ (except $\mathrm{C}^{17} \mathrm{O} 2 \rightarrow 1$, SO $3_{2} \rightarrow 2_{1}$ and SO $4_{3} \rightarrow 3_{2}$ to $20 \mathrm{~km} \mathrm{~s}^{-1}$ ). The intensity scale is $T_{\mathrm{A}}^{*}$ and the velocity scale is relative to the local standard of rest (l.s.r.) 

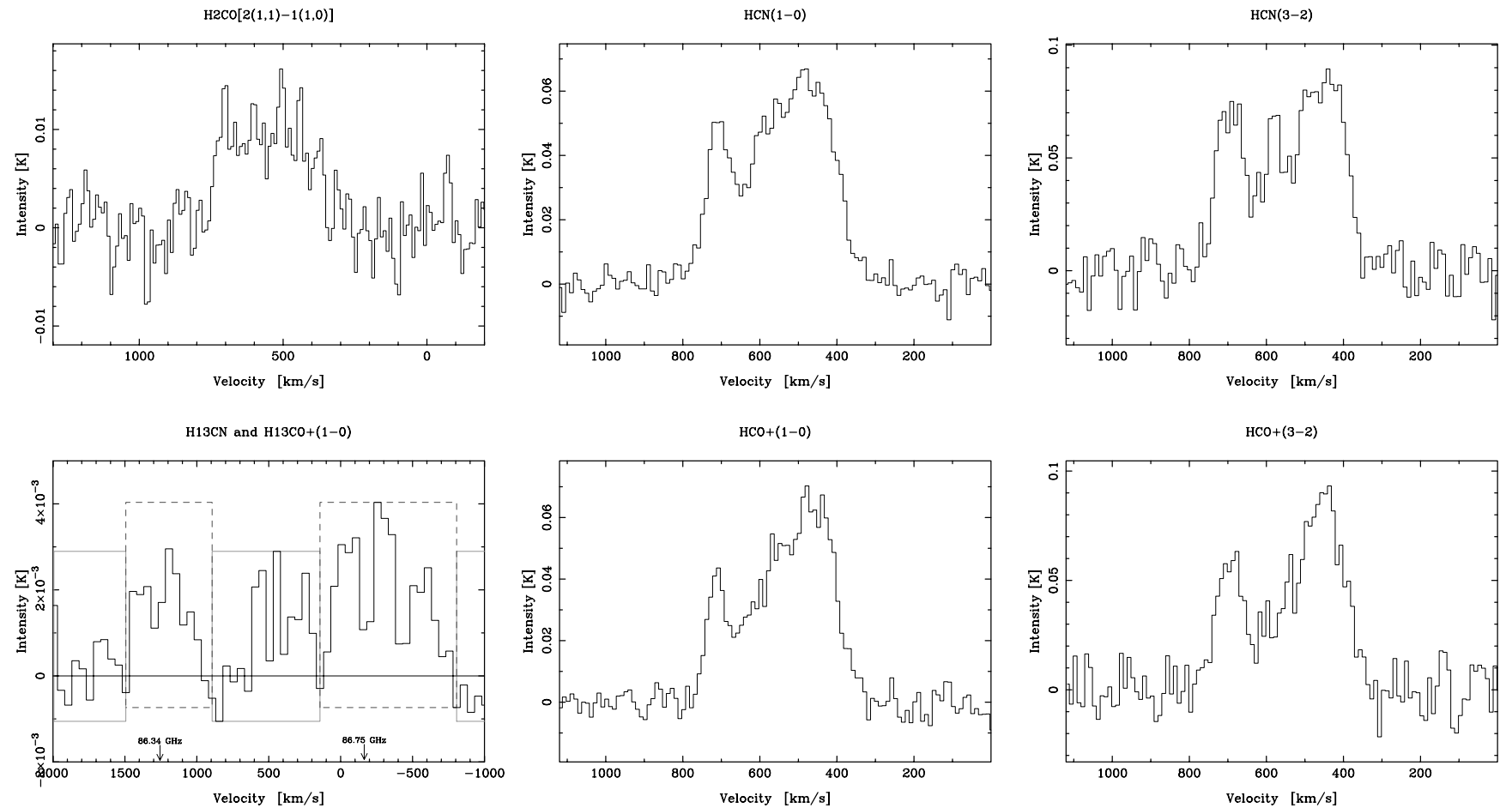

Fig. 1. continued. The $\mathrm{H}^{13} \mathrm{CN}$ and $\mathrm{H}^{13} \mathrm{CO}^{+} 1 \rightarrow 0$ transitions lie in the same band, centred on $86.55 \mathrm{GHz}$, at $86.34 \mathrm{GHz}$ $\left(1280 \mathrm{~km} \mathrm{~s}^{-1}\right)$ and $86.75 \mathrm{GHz}\left(-140 \mathrm{~km} \mathrm{~s}^{-1}\right)$, respectively. These spectra are shown with a resolution corresponding to $50 \mathrm{~km} \mathrm{~s}^{-1}$ (the remainder of the spectra being shown smoothed to $10 \mathrm{~km} \mathrm{~s}^{-1}$ ). The weak detections (here and in Fig. 2), are shown with the baseline and moment boxes used to determine their integrated intensities, given in Table 1

assumed source size) beam-filling has not been taken into account. Conversion to beam-averaged quantities is obtained by multiplication by $\eta_{\mathrm{bf}}$ (see Table 3 for the definition of this).

\subsubsection{The radiative transfer results}

The code used here is described in Jansen (1995): The excitation problem involves statistical equilibrium of a multilevel system (incorporating typically 12 rotational levels in the lowest vibrational state of the molecule in question). The radiative transfer is treated in the mean-escape probability (MEP) approximation: like the large velocity gradient (LVG) method (e.g. Leung \& Liszt 1976), this uses a local source function in which the optical depth in each transition determines the mean escape probability (Osterbrock 1989) of a photon from a typical location within a cloud. The model gas cloud has a spherical shape and a uniform density and kinetic temperature. The gas density and the temperature are estimated by fitting the observed line ratios of different transitions of $\mathrm{CO}, \mathrm{CS}$, $\mathrm{HCN}$ and $\mathrm{HCO}^{+}$to the excitation and radiative transfer model.

The intensity ratios for each species were constrained by a routine which tested the goodness-of-fit by calculating the $\chi^{2}$ error of the MEP integrated intensity ratios for each of the observed values. The ${ }^{12} \mathrm{CO}$ and $\mathrm{C}^{18} \mathrm{O}$ intensity ratios gave limits for the column density whereas ${ }^{13} \mathrm{CO}$ was quite specific (e.g. using $2 \rightarrow 1 / 1 \rightarrow 0=1.9$ in NGC 4945). Examining the results we selected, for example, the ${ }^{12} \mathrm{CO}$ column density which gave the observed ${ }^{12} \mathrm{CO} 1 \rightarrow 0$ to ${ }^{13} \mathrm{CO} 1 \rightarrow 0$ intensity ratio. This process was repeated for each transition of each isotopomer.

For NGC 4945 this gave $N\left({ }^{12} \mathrm{CO}\right) / N\left({ }^{13} \mathrm{CO}\right) \approx 50$ for the relative column densities and solutions could only be found for $T_{\mathrm{kin}} \approx 100 \mathrm{~K}$ and $n_{\mathrm{H}_{2}} \approx 310^{3} \mathrm{~cm}^{-3}$, i.e. as Henkel et al. (1994). Constraining the HCN solutions using the $1 \rightarrow 0 / 3 \rightarrow 2$ and the $\mathrm{CO} / \mathrm{HCN} 1 \rightarrow 0 / 1 \rightarrow 0$ and $3 \rightarrow 2 / 3 \rightarrow 2$ line ratios, we could obtain a solution for $n_{\mathrm{H}_{2}} \approx 10^{4}-10^{5} \mathrm{~cm}^{-3}$, regardless of kinetic temperature. For CS at the kinetic temperature defined by the $\mathrm{CO}$, the $2 \rightarrow 1 / 3 \rightarrow 2$ line ratios only permit a value of $n_{\mathrm{H}_{2}} \approx$ $10^{4} \mathrm{~cm}^{-3}$. Note that solutions at this molecular hydrogen density can be found over a range of kinetic temperatures (e.g. $T_{\text {kin }}=10 \mathrm{~K}, N_{\mathrm{CS}} / \Delta v \approx 610^{14} \mathrm{~cm}^{-2}\left(\mathrm{~km} \mathrm{~s}^{-1}\right)^{-1}$ and $\left.T_{\text {kin }}=150 \mathrm{~K}, N_{\mathrm{CS}} / \Delta v \approx 510^{13} \mathrm{~cm}^{-2}\left(\mathrm{~km} \mathrm{~s}^{-1}\right)^{-1}\right)$, but for densities higher than this $\left(n_{\mathrm{H}_{2}}=10^{5} \mathrm{~cm}^{-3}\right)$, solutions can only be found for $T_{\text {kin }} \lesssim 20 \mathrm{~K}$ (for $n_{\mathrm{H}_{2}}=10^{6} \mathrm{~cm}^{-3}$ there are no solutions). It is important to note that these results apply to the observed intensity ratios (within errors) only ${ }^{8}$, and so still hold if the CS traces a different gas component to the CO. The upper limit for the $1 \rightarrow 0 / 3 \rightarrow 2$ transitions of $\mathrm{H}^{13} \mathrm{CN}$ together with the $\mathrm{HCN} / \mathrm{H}^{13} \mathrm{CN} 1 \rightarrow 0 / 1 \rightarrow 0$ ratio gives the range of column densities shown in Table 5, where the MEP results

8 The CO/CS ratios have not been considered, although these are simultaneously satisfied. 

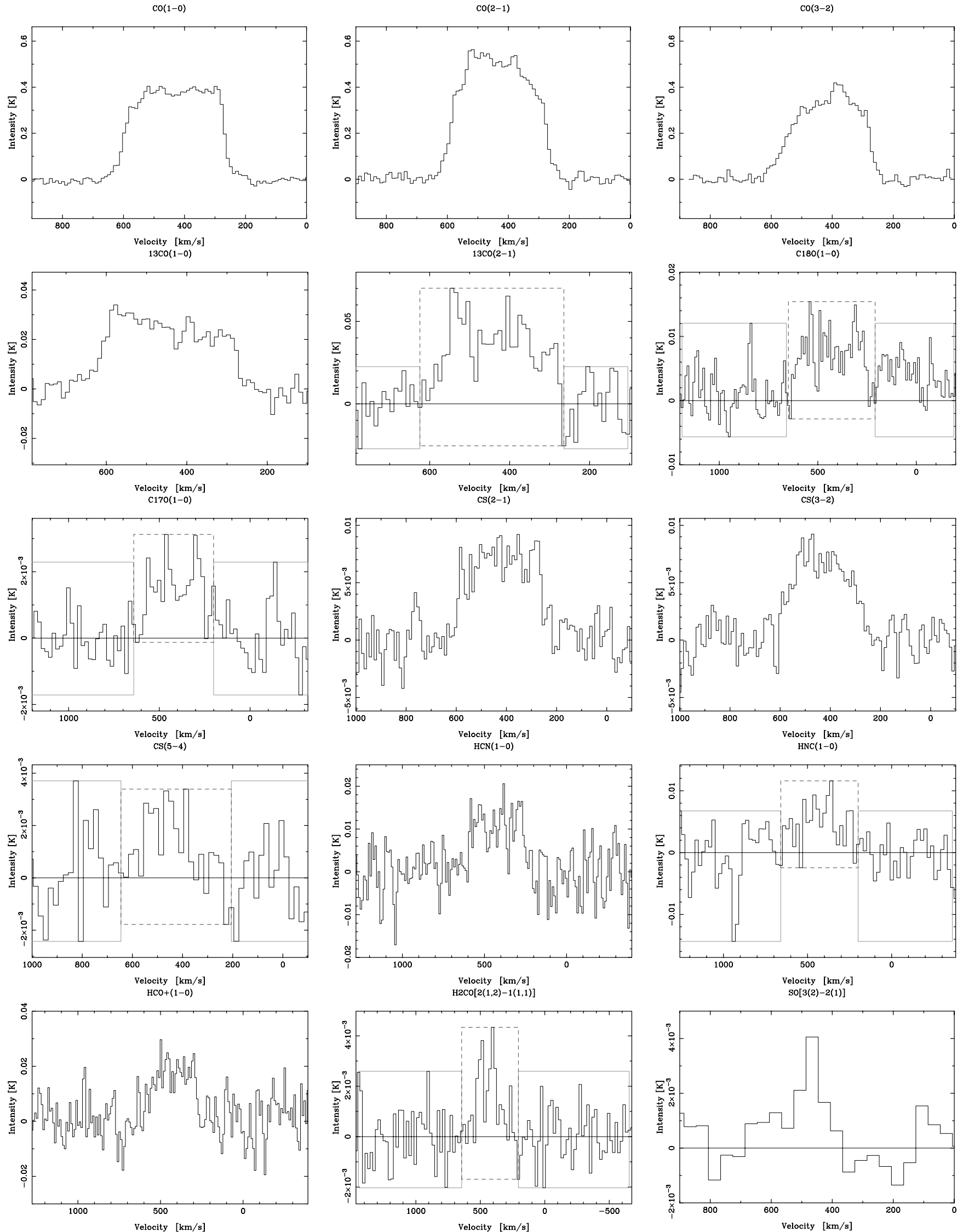

Fig. 2. The observed spectra in Circinus. A constant or first order baseline has been subtracted from each spectra. The spectra have been smoothed to a channel width corresponding to $10 \mathrm{~km} \mathrm{~s}^{-1}$ (except $\mathrm{C}^{17} \mathrm{O} 1 \rightarrow 0$, CS $5 \rightarrow 4, \mathrm{HNC} 1 \rightarrow 0$ and $\mathrm{H}_{2} \mathrm{CO}$ $\left(2_{1,2} \rightarrow 1_{1,1}\right)$ to $\left.20 \mathrm{~km} \mathrm{~s}^{-1}\right)$ and SO $3_{2} \rightarrow 2_{1}$ to $40 \mathrm{~km} \mathrm{~s}^{-1}$. The intensity scale is $T_{\mathrm{A}}^{*}$ and the velocity scale is relative to the local standard of rest (l.s.r.) 
Table 4. Integrated line intensity ratios. For the values listed in Table 3 the uncertainties in $\Theta_{\text {source }}$ (typically $10 \%$ ) lead to uncertainties of $\approx 10 \%$ in the values of $\eta_{\mathrm{bf}}\left(\frac{\Delta \eta}{\eta}=2(1-\eta) \frac{\Delta \Theta}{\Theta}\right)$, or $\sim 20 \%$ for ratios involving different source sizes

\begin{tabular}{|c|c|c|c|}
\hline \multirow[t]{2}{*}{ Molecule(s) } & \multirow[t]{2}{*}{ Transitions } & \multicolumn{2}{|c|}{$I$ ratio } \\
\hline & & NGC 4945 & Circinus \\
\hline \multirow[t]{2}{*}{${ }^{12} \mathrm{CO}$} & $2 \rightarrow 1 / 1 \rightarrow 0$ & $1.0 \pm 0.2$ & $1.0 \pm 0.2$ \\
\hline & $3 \rightarrow 2 / 1 \rightarrow 0$ & $0.9 \pm 0.2$ & $0.9 \pm 0.2$ \\
\hline${ }^{13} \mathrm{CO}$ & $2 \rightarrow 1 / 1 \rightarrow 0$ & $1.9 \pm 0.4$ & $1.5 \pm 0.5$ \\
\hline \multirow{2}{*}{${ }^{12} \mathrm{CO} /{ }^{13} \mathrm{CO}$} & $1 \rightarrow 0 / 1 \rightarrow 0$ & $17 \pm 2$ & $14 \pm 2$ \\
\hline & $2 \rightarrow 1 / 2 \rightarrow 1$ & $8.3 \pm 0.8$ & $10 \pm 3$ \\
\hline $\mathrm{C}^{18} \mathrm{O}$ & $2 \rightarrow 1 / 1 \rightarrow 0$ & $2.4 \pm 0.6$ & - \\
\hline \multirow[t]{2}{*}{${ }^{12} \mathrm{CO} / \mathrm{C}^{18} \mathrm{O}$} & $1 \rightarrow 0 / 1 \rightarrow 0$ & $59 \pm 9$ & $41 \pm 7$ \\
\hline & $2 \rightarrow 1 / 2 \rightarrow 1$ & $25 \pm 4$ & - \\
\hline $\mathrm{C}^{17} \mathrm{O}$ & $2 \rightarrow 1 / 1 \rightarrow 0$ & $1.7 \pm 0.9$ & - \\
\hline \multirow[t]{2}{*}{${ }^{12} \mathrm{CO} / \mathrm{C}^{17} \mathrm{O}$} & $1 \rightarrow 0 / 1 \rightarrow 0$ & $300 \pm 80$ & $230 \pm 50$ \\
\hline & $2 \rightarrow 1 / 2 \rightarrow 1$ & $170 \pm 50$ & - \\
\hline \multirow[t]{2}{*}{$\mathrm{C}^{18} \mathrm{O} / \mathrm{C}^{17} \mathrm{O}$} & $1 \rightarrow 0 / 1 \rightarrow 0$ & $5 \pm 2$ & $6 \pm 2$ \\
\hline & $2 \rightarrow 1 / 2 \rightarrow 1$ & $7 \pm 2$ & - \\
\hline \multirow[t]{2}{*}{ CS } & $2 \rightarrow 1 / 3 \rightarrow 2$ & $1.7 \pm 0.3$ & $1.8 \pm 0.6$ \\
\hline & $2 \rightarrow 1 / 5 \rightarrow 4$ & - & $7 \pm 3$ \\
\hline $\mathrm{CS} /{ }^{13} \mathrm{CS}$ & $2 \rightarrow 1 / 2 \rightarrow 1$ & $>4$ & - \\
\hline $\mathrm{SO}$ & $3_{2} \rightarrow 2_{1} / 4_{3} \rightarrow 3_{2}$ & $1.4 \pm 0.9$ & - \\
\hline \multirow[t]{2}{*}{$\mathrm{CS} / \mathrm{SO}$} & $2 \rightarrow 1 / 3_{2} \rightarrow 2_{1}$ & $8 \pm 3$ & $3 \pm 1$ \\
\hline & $3 \rightarrow 2 / 4_{3} \rightarrow 3_{2}$ & $7 \pm 3$ & - \\
\hline $\mathrm{HCN}$ & $1 \rightarrow 0 / 3 \rightarrow 2$ & $2.6 \pm 0.4$ & - \\
\hline $\mathrm{HCN} / \mathrm{H}^{13} \mathrm{CN}$ & $1 \rightarrow 0 / 1 \rightarrow 0$ & $20 \pm 10$ & - \\
\hline $\mathrm{HCN} / \mathrm{HNC}$ & $1 \rightarrow 0 / 1 \rightarrow 0$ & - & $1.7 \pm 0.8$ \\
\hline $\mathrm{HCO}^{+}$ & $1 \rightarrow 0 / 3 \rightarrow 2$ & $2.7 \pm 0.4$ & - \\
\hline \multirow[t]{2}{*}{${ }^{12} \mathrm{CO} / \mathrm{CS}$} & $2 \rightarrow 1 / 2 \rightarrow 1$ & $23 \pm 5$ & $31 \pm 6$ \\
\hline & $3 \rightarrow 2 / 3 \rightarrow 2$ & $36 \pm 5$ & $52 \pm 10$ \\
\hline \multirow[t]{2}{*}{${ }^{12} \mathrm{CO} / \mathrm{HCN}$} & $1 \rightarrow 0 / 1 \rightarrow 0$ & $7.5 \pm 0.9$ & $17 \pm 4$ \\
\hline & $3 \rightarrow 2 / 3 \rightarrow 2$ & $18 \pm 3$ & - \\
\hline \multirow[t]{2}{*}{${ }^{12} \mathrm{CO} / \mathrm{HCO}^{+}$} & $1 \rightarrow 0 / 1 \rightarrow 0$ & $8 \pm 1$ & $13 \pm 4$ \\
\hline & $3 \rightarrow 2 / 3 \rightarrow 2$ & $20 \pm 3$ & - \\
\hline \multirow[t]{2}{*}{$\mathrm{HCN} / \mathrm{HCO}^{+}$} & $1 \rightarrow 0 / 1 \rightarrow 0$ & $1.2 \pm 0.2$ & $0.7 \pm 0.2$ \\
\hline & $3 \rightarrow 2 / 3 \rightarrow 2$ & $1.2 \pm 0.2$ & - \\
\hline $\mathrm{HCO}^{+} / \mathrm{H}^{13} \mathrm{CO}^{+}$ & $1 \rightarrow 0 / 1 \rightarrow 0$ & $9 \pm 3$ & - \\
\hline $\mathrm{HCO}^{+} /{ }^{13} \mathrm{CO}$ & $1 \rightarrow 0 / 1 \rightarrow 0$ & $2.0 \pm 0.2$ & $1.2 \pm 0.3$ \\
\hline $\mathrm{HCO}^{+} / \mathrm{CS}$ & $3 \rightarrow 2 / 3 \rightarrow 2$ & $1.7 \pm 0.3$ & - \\
\hline $\mathrm{H}^{13} \mathrm{CN}$ & $1 \rightarrow 0 / 3 \rightarrow 2$ & $>3$ & - \\
\hline $\mathrm{H}_{2} \mathrm{CO}$ & $\frac{2_{1,2} \rightarrow 1_{1,1}+2_{1,1} \rightarrow 1_{1,0}}{2_{0,2} \rightarrow 1_{0,1}}$ & $2.3 \pm 0.7$ & - \\
\hline $\mathrm{H}_{2} \mathrm{CO}$ & $2_{1,2} \rightarrow 1_{1,1} / 2_{1,1} \rightarrow 1_{1,0}$ & $1.2 \pm 0.2$ & - \\
\hline $\mathrm{H}_{2} \mathrm{CO}$ & $2_{1,2} \rightarrow 1_{1,1} / 2_{0,2} \rightarrow 1_{0,1}$ & $1.3 \pm 0.2$ & - \\
\hline
\end{tabular}

are summarised. Note that, as for the CO isotopomers, we obtain a $\mathrm{HCN} / \mathrm{H}^{13} \mathrm{CN}$ column density ratio of $\approx 50-200$.

For the $\mathrm{CO}$ values in Circinus, solutions could only be found for $T_{\text {kin }} \approx 50-80 \mathrm{~K}$ and $n_{\mathrm{H}_{2}}=210^{3} \mathrm{~cm}^{-3}$, i.e. as the warm gas solution of Curran et al. (1998), where we obtained solutions of the CO isotopomers convolved to the ${ }^{12} \mathrm{CO} 1 \rightarrow 0$ beam. The column densities give $N\left({ }^{12} \mathrm{CO}\right) / N\left({ }^{13} \mathrm{CO}\right)=60-80$. Since we have only the $1 \rightarrow 0$ transition available in this galaxy, models for
$\mathrm{HCN}$ and $\mathrm{HCO}^{+}$are not so easy to constrain. Assuming, however, a similar kinetic temperature between the various tracers and using the ${ }^{12} \mathrm{CO} / \mathrm{HCN} 1 \rightarrow 0 / 1 \rightarrow 0$ and ${ }^{12} \mathrm{CO} / \mathrm{HCO}^{+} 1 \rightarrow 0 / 1 \rightarrow 0$ ratios (i.e. the $\mathrm{HCN}$ and $\mathrm{CO}$ trace similar regions), solutions may be found for $n_{\mathrm{H}_{2}} \sim 10^{4}-10^{6} \mathrm{~cm}^{-3}$. From the $2 \rightarrow 1 / 3 \rightarrow 2$ and $2 \rightarrow 1 / 5 \rightarrow 4$ ratios of CS, we could only find a solution at a molecular hydrogen density of $n_{\mathrm{H}_{2}} \sim 10^{5} \mathrm{~cm}^{-3}$. Note that solutions using this molecule could only be 

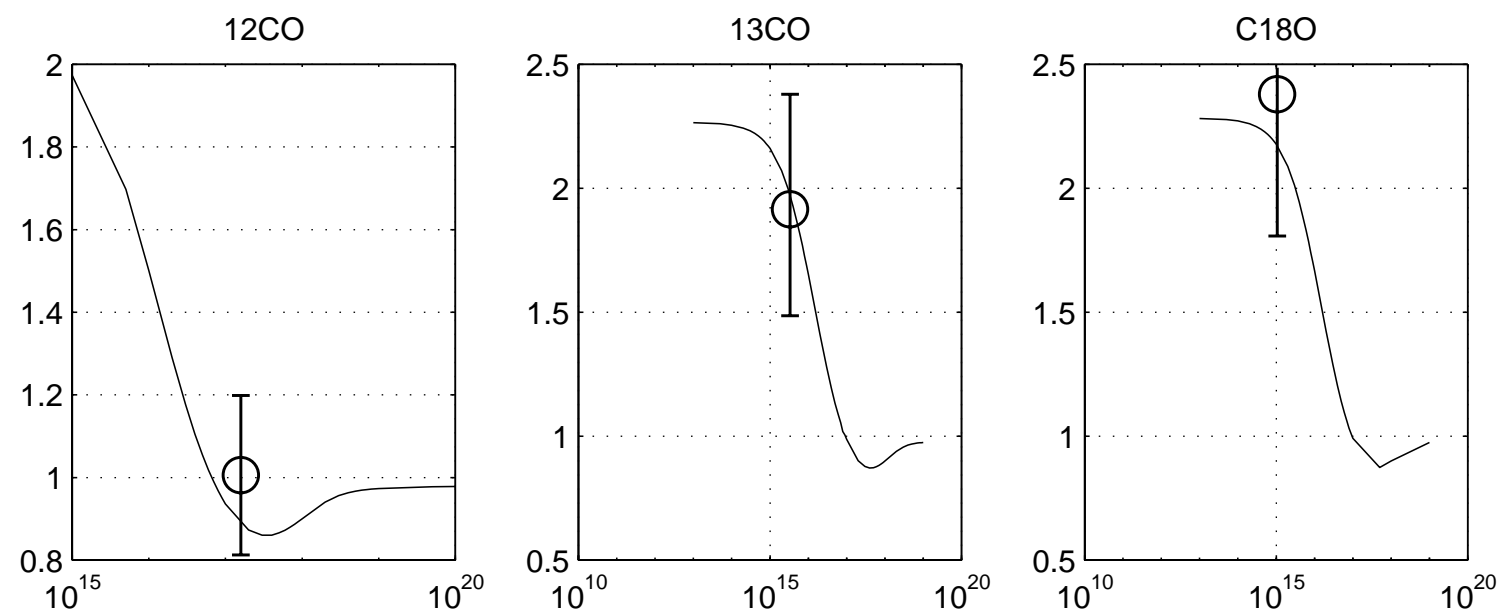

Fig. 3. MEP solutions for the CO isotopomers per velocity interval at $T_{\text {kin }}=100 \mathrm{~K}, n_{\mathrm{H}_{2}}=310^{3} \mathrm{~cm}^{-3}$ (i.e. for NGC 4945 ). The plots show the $2 \rightarrow 1 / 1 \rightarrow 0$ intensity ratio against the column density per unit line width $\left[\mathrm{cm}^{-2}\left(\mathrm{~km} \mathrm{~s}^{-1}\right)^{-1}\right]$ for each isotopomer, and give $N\left({ }^{12} \mathrm{CO}\right) / \Delta v \gtrsim 310^{16} \mathrm{~cm}^{-2}\left(\mathrm{~km} \mathrm{~s}^{-1}\right)^{-1},\left(N^{13} \mathrm{CO}\right) / \Delta v \sim 10^{16} \mathrm{~cm}^{-2}\left(\mathrm{~km} \mathrm{~s}^{-1}\right)^{-1}$ and $N\left(\mathrm{C}^{18} O\right) / \Delta v \lesssim$ $10^{16} \mathrm{~cm}^{-2}\left(\mathrm{~km} \mathrm{~s}^{-1}\right)^{-1}$. In this and Fig. 4, the circles indicate the nominal observed values with the associated uncertainties shown as bars. Note that these are only the solutions for each isotopomer and are therefore valid over the range of column densities constrained by the error bars. In the case of the final solutions (Tables 5 and 6 ), however, we also take into account the ratios between different isotopomers

Table 5. The MEP solutions for NGC 4945 for $T_{\text {kin }}=100 \mathrm{~K}$. The column density is derived for a cloud velocity width of $30 \mathrm{~km} \mathrm{~s}^{-1}$, Eq. (1). These solutions satisfy both the observed line ratios for each isotopomer and the line ratios between isotopomers (within uncertainties)

\begin{tabular}{lcr}
\hline Molecule & $n_{\mathrm{H}_{2}}\left[\mathrm{~cm}^{-3}\right]$ & $N\left[\mathrm{~cm}^{-2}\right]$ \\
\hline${ }^{12} \mathrm{CO}$ & $310^{3}$ & $\approx 610^{18}$ \\
${ }^{13} \mathrm{CO}$ & $310^{3}$ & $1.2-1.510^{17}$ \\
$\mathrm{C}^{18} \mathrm{O}$ & $310^{3}$ & $\approx 310^{16}$ \\
$\mathrm{HCN}$ & $10^{4}$ & $1.5-2.410^{16}$ \\
& $10^{5}$ & $\approx 310^{15}$ \\
$\mathrm{CS}$ & $10^{4}$ & $1.5-2.410^{15}$ \\
$\mathrm{HCO}+$ & $10^{4}$ & $1.5-2.110^{15}$ \\
$\mathrm{H}^{13} \mathrm{CN}$ & $10^{4}$ & $1-310^{14}$ \\
\hline
\end{tabular}

obtained over a similar temperature range as for the CO. No solutions which satisfy the observed CO/CS ratios at $n_{\mathrm{H}_{2}} \approx 10^{4}$ or $10^{6} \mathrm{~cm}^{-3}$ could be found, regardless of kinetic temperature ${ }^{9}$. The results are summarised in Table 6 .

\footnotetext{
9 Solutions for the isotopomer intensity ratios only could be found at the lower end of this range i.e. for $n_{\mathrm{H}_{2}} \approx$ $10^{4}-10^{5} \mathrm{~cm}^{-3}$ (but only for $T_{\text {kin }} \approx 100 \mathrm{~K}$ ). However, unlike $n_{\mathrm{H}_{2}} \approx 10^{5} \mathrm{~cm}^{-3}$, applying a molecular hydrogen density of $n_{\mathrm{H}_{2}} \approx 10^{4} \mathrm{~cm}^{-3}$ could not satisfy the observed CO/CS ratios. Such a result could be feasible if the CS traced a different component of the gas, but we consider it unlikely that this would have such a similar (low) density and kinetic temperature.
}

Table 6. The MEP solutions for Circinus for $T_{\text {kin }}=50-80 \mathrm{~K}$. The column density is derived for a cloud velocity width of $10 \mathrm{~km} \mathrm{~s}^{-1}$, Eq. (1). These solutions satisfy both the observed line ratios for each isotopomer and the line ratios between isotopomers (within uncertainties)

\begin{tabular}{lcr}
\hline Molecule & $n_{\mathrm{H}_{2}}\left[\mathrm{~cm}^{-3}\right]$ & $N\left[\mathrm{~cm}^{-2}\right]$ \\
\hline${ }^{12} \mathrm{CO}$ & $210^{3}$ & $\approx 210^{18}$ \\
${ }^{13} \mathrm{CO}$ & $210^{3}$ & $2.5-310^{16}$ \\
$\mathrm{HCN}$ & $10^{4}$ & $\approx 110^{15}$ \\
& $10^{5}$ & $\approx 110^{14}$ \\
& $10^{6}$ & $<110^{14}$ \\
$\mathrm{CS}$ & $10^{5}$ & $\approx 510^{13}$ \\
$\mathrm{HCO}^{+}$ & $10^{5}$ & $\approx 310^{13}$ \\
\hline
\end{tabular}

Using the total observed line emission to estimate an individual cloud velocity width (neglecting effects due to cloud-cloud shielding) from

$\Delta v=\left(1+\frac{\Theta_{\mathrm{mb}}^{2}}{\Theta_{\text {source }}^{2}}\right) \sqrt{4 \ln 2 / \pi} \frac{I_{\mathrm{mb}}}{T_{\mathrm{MEP}}}$,

where $I_{\mathrm{mb}}$ is the total velocity integrated intensity (Tables 1 and 2) and $T_{\mathrm{MEP}}$ is the antenna temperature obtained from the MEP analysis ${ }^{10}$, we find velocity widths of $\approx 30 \mathrm{~km} \mathrm{~s}^{-1}$ and $\approx 10 \mathrm{~km} \mathrm{~s}^{-1}$ for NGC 4945 and Circinus, respectively. These values are applied to the derived column densities per unit line width to give the values shown in Tables 5 and 6.

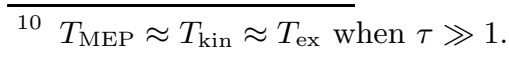


$12 \mathrm{CO}$

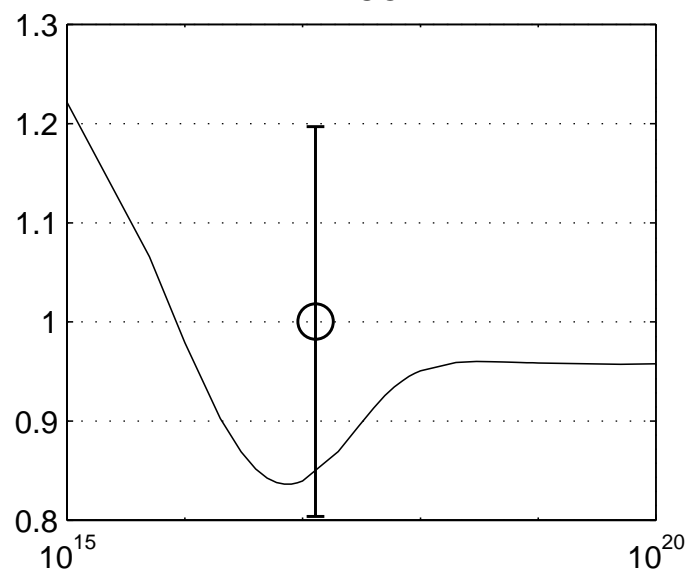

$13 \mathrm{CO}$

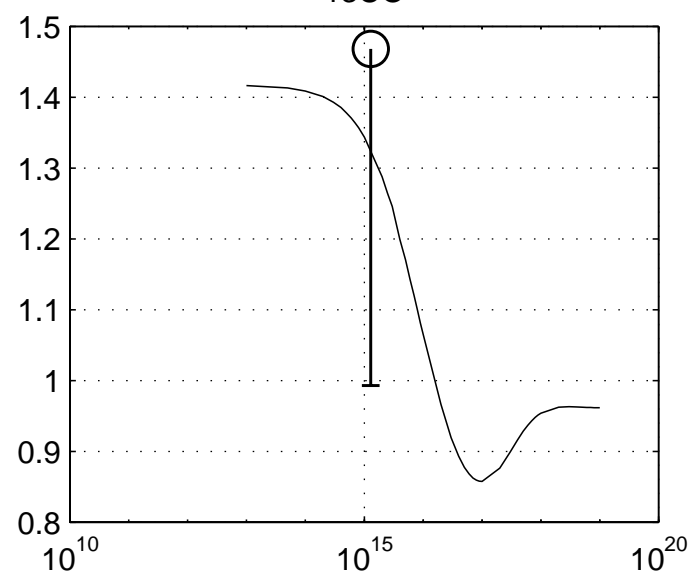

Fig. 4. MEP solutions for the CO isotopomers per velocity interval at $T_{\text {kin }}=50 \mathrm{~K}, n_{\mathrm{H}_{2}}=210^{3} \mathrm{~cm}^{-3}$ (i.e. for Circinus). The plots show the $2 \rightarrow 1 / 1 \rightarrow 0$ intensity ratio against the column density per unit line width $\left[\mathrm{cm}^{-2}\left(\mathrm{~km} \mathrm{~s}^{-1}\right)^{-1}\right]$ for each isotopomer, and give $N\left({ }^{12} \mathrm{CO}\right) / \Delta v \gtrsim 10^{15} \mathrm{~cm}^{-2}\left(\mathrm{~km} \mathrm{~s}^{-1}\right)^{-1}$ and $N\left({ }^{13} \mathrm{CO}\right) / \Delta v \lesssim 10^{16} \mathrm{~cm}^{-2}\left(\mathrm{~km} \mathrm{~s}^{-1}\right)^{-1}$

Table 7. The minimum $\chi^{2}$ LTE solutions. Note that, unlike previously, these are beam averaged column densities (based upon the velocity integrated intensities)

\begin{tabular}{lccc}
\hline Galaxy & $T_{\text {ex }}[\mathrm{K}]$ & $N\left({ }^{12} \mathrm{CO}\right)\left[\mathrm{cm}^{-2}\right]$ & $N\left({ }^{12} \mathrm{CO}\right) / N\left({ }^{13} \mathrm{CO}\right)$ \\
\hline NGC 4945 & 20 & $210^{19}$ & 40 \\
Circinus & 14 & $210^{18}$ & 25 \\
\hline
\end{tabular}

\subsubsection{The LTE results}

In order to complement the MEP results, we applied the LTE model to the observed data. Since this method ${ }^{11}$ depends only upon the excitation temperature $\left(T_{\mathrm{ex}}\right)$ and the column density, it is the simplest way to analyse the observed line ratios. Here we have assumed that the excitation temperature is the same for both ${ }^{12} \mathrm{CO}$ and ${ }^{13} \mathrm{CO}$.

From the solutions (Table 7) we see that the LTE results differ significantly from the solutions obtained from the radiative transfer calculations, although the derived column densities are reasonable, cf. $\approx 810^{19} \mathrm{~cm}^{-2}$ for the peak column densities in NGC 4945, obtained by multiplying the ${ }^{12} \mathrm{CO}$ column density per unit line width $\left(\approx 210^{17} \mathrm{~cm}^{-2}\left(\mathrm{~km} \mathrm{~s}^{-1}\right)^{-1}\right)$ over the full line width (Fig. 1). For Circinus the value obtained is closer to that for an individual cloud (see Table 6). Applying the temperatures and column densities from the LTE solutions to the MEP code we can satisfy the observed intensity ratios for $N\left({ }^{12} \mathrm{CO}\right)>610^{19} \mathrm{~cm}^{-2}$ and $>510^{19} \mathrm{~cm}^{-2}$ in NGC 4945 and Circinus, respectively. We cannot, however, reproduce the observed ${ }^{13} \mathrm{CO}$ ratios: ${ }^{13} \mathrm{CO} 2 \rightarrow$ $1 / 1 \rightarrow 0<1.1$ for NGC 4945 and $<0.87$ (or $\approx 0.9$ for $\left.N\left({ }^{13} \mathrm{CO}\right)>210^{18} \mathrm{~cm}^{-2}\right)$ for Circinus using the molecular hydrogen densities obtained from the MEP solutions.

\footnotetext{
11 A description of the LTE method is given in e.g. Turner (1991).
}

\section{Discussion}

From the MEP analysis it appears that the molecular gas in the two galaxies arises from a relatively dense phase, i.e. $n_{\mathrm{H}_{2}}=310^{3}-10^{4} \mathrm{~cm}^{-3}$ in NGC 4945 and $n_{\mathrm{H}_{2}}=210^{3}-10^{5} \mathrm{~cm}^{-3}$ in Circinus ${ }^{12}$. The observed line ratios may be solved for a constant kinetic temperature in each galaxy, i.e. $T_{\text {kin }}=100 \mathrm{~K}$ and $T_{\text {kin }}=50-80 \mathrm{~K}$ in NGC 4945 and Circinus, respectively. Our results for NGC 4945 compare well with those of the LVG model of Henkel et al. (1994), i.e. $T_{\text {kin }}=100 \mathrm{~K}, n_{\mathrm{H}_{2}}=310^{3} \mathrm{~cm}^{-3}$ and ${ }^{12} \mathrm{C} /{ }^{13} \mathrm{C} \sim 50$ for the $\mathrm{CO}$ cloud component. Like Henkel et al. (1994), we find the model of Bergman et al. (1992) insufficient in density, due to an underestimate of the ${ }^{12} \mathrm{CO} 2 \rightarrow 1 / 1 \rightarrow 0$ ratio (obtained from Whiteoak et al. 1990), in order to reproduce our value for this intensity ratio (which is similar to that previously obtained by Dahlem et al. 1993; Henkel et al. 1994). In the remainder of this section we will discuss the MEP results.

In NGC 4945 the star-burst is believed to have reached an advanced stage of evolution (Koornneef 1993; Henkel et al. 1994) and the presence of strong far-infrared and continuum radiation sources, as well as the water masers, indicate current vigorous star-formation activity (Moorwood \& Glass 1984; Moorwood \& Oliva 1994; Nakai et al. 1995; Moorwood et al. 1996b). Recent enrichment of the interstellar medium by massive stars is possibly indicated by a high ${ }^{18} \mathrm{O} /{ }^{17} \mathrm{O}$ abundance ratio. ${ }^{18} \mathrm{O}$ is believed to be produced by helium burning (i.e. in massive $\left(\gtrsim 10 M_{\odot}\right)$ stars, where the necessarily high temperatures of $\gtrsim 10^{8} \mathrm{~K}$ are found), whereas ${ }^{17} \mathrm{O}$ (the result of hydrogen burning, $\lesssim 10^{8} \mathrm{~K}$ ) originates in intermediate mass $\left(\lesssim 10 M_{\odot}\right)$ stars. Accordingly the ${ }^{18} \mathrm{O} /{ }^{17} \mathrm{O}$ abundance ratio may yield information on the relative abundances of

\footnotetext{
12 These values are arrived at by taking all isotopomer and inter-molecule ratios into account, i.e. $\mathrm{CO}, \mathrm{HCN}$ and $\mathrm{CS}$, of which the latter molecule constrains the upper value of $n_{\mathrm{H}_{2}}$ (previous section).
} 
these stars ${ }^{13}$. Estimating this from the $\mathrm{C}^{18} \mathrm{O} / \mathrm{C}^{17} \mathrm{O}$ intensity ratio, we obtain a value of $\approx 6$ in both NGC 4945 and Circinus. This compares well with the previously obtained value of Henkel et al. (1994); $8 \pm 2$. Also we obtain $\approx 30-60$ for the $\mathrm{C}^{16} \mathrm{O} / \mathrm{C}^{18} \mathrm{O}$ intensity ratio in NGC 4945 (cf. the value of $\approx 40$, Henkel et al. 1994) and the MEP analysis supports a column density ratio of $\approx 200$ (cf. $\approx 150$, Henkel et al. 1994). For Circinus the intensity ratio is $\approx 40$.

Looking at the $\mathrm{CO} / \mathrm{HCN}$ ratios, we see that ${ }^{12} \mathrm{CO}$ $1 \rightarrow 0 / \mathrm{HCN} 1 \rightarrow 0 \approx 8$ and $\approx 17$ for NGC 4945 and Circinus, respectively. Whereas the value for Circinus is similar to that expected from $L_{\mathrm{FIR}} \sim 10^{10} L_{\odot}$ Seyfert galaxies, the value for NGC 4945 is closer to that expected from $L_{\mathrm{FIR}} \sim 10^{11} L_{\odot}$ Seyferts (Curran et al. 2000). Naturally, since these two galaxies are somewhat closer than those of Curran et al. (2000), due to CO contamination from the disk, we would expect a lower $\mathrm{CO} / \mathrm{HCN}$ ratio provided that the $\mathrm{HCN}$ is more concentrated towards the nucleus than the $\mathrm{CO}$. The fact remains, however, that the $\mathrm{CO} / \mathrm{HCN}$ ratio in NGC 4945 is about half that in Circinus. If the CO traces the dynamical mass, and since both galaxies have a dynamical mass of $310^{9} M_{\odot}$ within the central 600 pc (Mauersberger et al. 1996; Curran et al. 1998), this suggests that there is twice as much HCN in the nuclear region of NGC $4945^{14}$.

Examining the HCN/FIR luminosity ratios, we find that for NGC 4945, $L_{\mathrm{HCN}}=19 \pm 1 \mathrm{~K} \mathrm{~km} \mathrm{~s}^{-1} \mathrm{kpc}^{2}$ and $L_{\mathrm{FIR}}=9.110^{9} L_{\odot}$ and that for Circinus $L_{\mathrm{HCN}}=$ $4.9 \pm 0.7 \mathrm{~K} \mathrm{~km} \mathrm{~s}^{-1} \mathrm{kpc}^{2}$ (the HCN in both galaxies within the HPBW of $57^{\prime \prime}$ ) and $L_{\mathrm{FIR}}=6.210^{9} L_{\odot}$ (calculated from Lonsdale et al. $1985^{15}$ ). These values give $\frac{L_{\mathrm{HCN}}}{L_{\mathrm{FIR}}}(\mathrm{NGC} 4945) \approx 2.5 \frac{L_{\mathrm{HCN}}}{L_{\mathrm{FIR}}}$ (Circinus) $)^{16}$, and this strongly suggests that the HCN luminosity in NGC 4945 arises from an additional component to the star forming cores, e.g. the dense gas component of the inner circumnuclear disk (Kohno et al. 1999; Curran et al. 2000): although the maser emission occurs on very small (pc) scales, the inner molecular gas structure ${ }^{17}$ which has an

\footnotetext{
13 See Prantzos et al. (1996) for a review.

14 Based upon the equivalent central dynamical masses of the two galaxies and assuming the same $N_{\mathrm{H}_{2}} / I_{\mathrm{CO}}$ conversion factor (Mauersberger et al. 1996; Curran et al. 1998).

15 Assuming distances of 3.7 Mpc (Mauersberger et al. 1996) and 4.0 Mpc (Freeman et al. 1977) to NGC 4945 and Circinus, respectively. Note that this results in a $4 \%$ increase in beam area over Circinus which is not sufficient to give a similar $\mathrm{HCN} / \mathrm{CO}$ ratio as NGC 4945.

${ }^{16}$ NGC 4945 lies closer to the line defined by the Seyfert sample (Fig. 7 of Curran et al. 2000), and while an incomplete sampling of the HCN will affect the ratios in both galaxies, the relative ratios are expected to remain similar. Note also that distance estimates to NGC 4945 vary somewhat, e.g. $6.7 \mathrm{Mpc}$ according to Dahlem et al. (1993); Forbes \& Norris (1998), this, however, has no effect on $\frac{L_{\mathrm{HCN}}}{L_{\mathrm{FIR}}}$.

17 Located well within the molecular ring, which occurs at the inner Lindblad resonance. See Curran (2000) for a review.
}

elevated (cf. the ring) $\mathrm{HCN}$ to $\mathrm{CO}$ intensity ratio ${ }^{18}$ is still extended enough to be perfectly detectable with beams of $\approx 2^{\prime \prime}$ in near-by AGNs (Sternberg et al. 1994; Helfer \& Blitz 1995; Tacconi et al. 1998). Note also that from mid-IR spectroscopy of NGC 4945, Spoon et al. (2000) postulate that compared to Circinus the narrow line region and/or UV radiation from the AGN may be severly obscured. This could perhaps be due to the central accumulation of dense gas and this hypothesis may be further supported by:

1. The results of Forbes \& Norris (1998) who find that the radio/FIR ratio is higher for NGC 4945 (in the radioloud AGN regime) than for the rest of their Seyfert sample (including Circinus), which share similar ratios to star-burst galaxies. They do, however, attribute this to an underestimate of the total FIR flux due to the source not being wholly sampled (Rice et al. 1988). This would lower the HCN/FIR luminosity ratio in NGC 4945 by a factor of 6 . It should be noted that Circinus and NGC 4945 (e.g. Forbes \& Norris 1998) are by far the two closest Seyferts, and due to its large extent (Freeman et al. 1977; Jones et al. 1999) a correction should also be required for Circinus, but this galaxy has not been included in the sample of Rice et al. (1988). This may have the effect of maintaining a similar radio/FIR relation between the two galaxies.

2. Although fairly dense at $10^{4} \mathrm{~cm}^{-3}$, the higher density tracers are still an order of magnitude more diffuse than in Circinus (based on the more definite CS value). Densities of $10^{5} \mathrm{~cm}^{-3}$ are typical of star-forming cloud cores (Solomon et al. 1990). Note also, from near-IR spectroscopy, Storchi-Bergmann et al. (1999) find that the nuclear excitation in Circinus is dominated by the star-burst activity.

The latter result contradicts the inferred star formation rates of $7.8 M_{\odot} \mathrm{yr}^{-1}$ for NGC 4945 (Dahlem et al. 1993) ${ }^{19}$ and 2.5 $M_{\odot} \mathrm{yr}^{-1}$ for Circinus (Elmouttie et al. 1998), but these estimates are based upon the total FIR luminosity which could be misleading if some of this radiation originates from the AGN.

In support of our excitation analysis, in Circinus we find $\operatorname{HNC}(1 \rightarrow 0) / \mathrm{HCN}(1 \rightarrow 0)=0.6 \pm 0.3$, which is typical of galaxies with $L_{\mathrm{IR}} \gtrsim 10^{9} L_{\odot}$ and suggests the presence of warm gas (Hüttemeister et al. 1995). In NGC 4945, assuming optically thin lines, we calculate a formaldehyde ortho/para ratio of $\mathrm{H}_{2} \mathrm{CO}\left(\frac{2_{1,2} \rightarrow 1_{1,1}+2_{1,1} \rightarrow 1_{1,0}}{2_{0,2} \rightarrow 1_{0,1}}\right) \approx$ 2.3 ; or probably even slightly higher than this since the

\footnotetext{
18 For example, from high resolution observations of NGC 1068, Helfer \& Blitz (1993); Helfer \& Blitz (1995) find that while the CO traces the star-burst ring and the more extended emission, most of the HCN is located in the nucleus with only a relatively small portion located in the ring. Tacconi et al. (1994) also find the HCN to be distributed in warm (>70 K) clouds, in such a fashion.

19 They consider this rate to be high compared with other star-burst galaxies.
} 
$2_{0,2} \rightarrow 1_{0,1}$ line is blended with the $\mathrm{HC}_{3} \mathrm{~N} 16 \rightarrow 15$ line $^{20}$, Table 1.

From HCN observations of the star-burst galaxies M 82 and NGC 253, which like NGC 4945 and Circinus share similar CO and FIR properties, Jackson et al. (1995) find that the $4 \rightarrow 3 / 1 \rightarrow 0$ intensity ratio gives molecular hydrogen and column densities per unit line width:

1. In M 82 the values are $\sim 10^{4} \quad \mathrm{~cm}^{-3}$ and $\sim 10^{14} \mathrm{~cm}^{-2}\left(\mathrm{~km} \mathrm{~s}^{-1}\right)^{-1}$, respectively, i.e. similar to what we find in NGC 4945;

2. In NGC 253 the values are $\sim 10^{5} \mathrm{~cm}^{-3}$ and $\sim 10^{13} \mathrm{~cm}^{-2}\left(\mathrm{~km} \mathrm{~s}^{-1}\right)^{-1}$, respectively, i.e. similar to what we find in Circinus.

That is, gas densities of an order of magnitude greater, within $300 \mathrm{pc}^{21}$ (where the gas is densest), in NGC 253 than in M 82. Although both are classed as star-burst galaxies, from the literature M 82 appears to be fairly typical with a $100-$ pc scale star-burst ring (e.g. Young \& Scoville 1982; Nakai et al. 1987; Curran et al. 1998), but possibly harbouring a weak AGN (Wills et al. 1999), while the centre of NGC 253 appears to be completely dominated by star forming activity, i.e. a large number of individual radio and X-ray sources due to the presence of stars (i.e. formation, supernova remnants and X-ray binaries) located within the innermost $\sim 20 \mathrm{pc}$ of the nucleus (Ulvestad \& Antonucci 1997; Vogler \& Pietsch 1999). And so the conditions in M 82 and NGC 253, may be likened to those in NGC 4945 and Circinus, respectively, may possibly reflect the presence/significant contribution of an AGN, i.e. the higher density gas in Circinus and NGC 253 being due to a larger fraction of the radiation arising from star formation cf. NGC 4945 and M $82^{22}$. For these two galaxies there also exist observations of the CS $2 \rightarrow 1$ and SO $3_{2} \rightarrow 2_{1}$ transitions. These are excited collisionally under similar conditions ${ }^{23}$ (e.g. Petuchowski $\&$ Bennett 1992) and so prove useful in probing the conditions of the molecular gas. For the CS $2 \rightarrow 1$ to SO $3_{2} \rightarrow 2_{1}$ intensity ratio we obtain a value of $8 \pm 3$ in

\footnotetext{
${ }^{20}$ In the star-burst galaxy NGC 253 the $\mathrm{H}_{2}$ ortho/para ratio is $\sim 2$ (Harrison et al. 1998), corresponding to densities and temperatures of $\sim 10^{5} \mathrm{~cm}^{-3}$ and $\approx 50-100 \mathrm{~K}$, respectively (next paragraph).

21 Where our higher transitions probe in NGC 4945 and Circinus.

${ }^{22}$ Applying our total integrated intensity for $\mathrm{HCN} 1 \rightarrow 0$ (which is identical to the value of Henkel et al. 1990 used by Jackson et al. 1995), gives a $4 \rightarrow 3 / 1 \rightarrow 0$ intensity ratio (of $\approx 0.35$ ) for NGC 4945 which is greater than that of M 82 but less than that of NGC 253, perhaps indicating the more prominent AGN.

${ }^{23}$ The reason for this is that they have similar A-coefficients and state energies. The intensity ratio which is insensitive to density and temperature variations, should therefore be mainly dependent on the ratio of the column densities. In fact, due to the 3 -fold spin multiplicity of SO, a CS $2 \rightarrow 1$ to SO $3_{2} \rightarrow$ 21 intensity ratio ratio of 3 implies similar column densities; $N(\mathrm{SO}) \approx N(\mathrm{CS})$.
}

NGC 4945 and $3 \pm 1$ in Circinus; the former value agreeing with those of both M 82 and NGC 253, i.e. $\approx 8$ and $\approx 7$, respectively $^{24}$ (Sage et al. 1990; Petuchowski \& Bennett 1992). Also, Galactic GMC data (Nilsson et al. 2000) show $\mathrm{CS} 2 \rightarrow 1 / \mathrm{SO} 3_{2} \rightarrow 2_{1}$ intensity ratios in the range $0.3-11$, and typical ${ }^{25}$ values are in the range $2-5$, i.e. as for Circinus $(N(\mathrm{SO}) \approx N(\mathrm{CS}))^{26}$, while $\mathrm{SO}$ seems to be a factor of 2-3 less abundant than CS in NGC 4945.

\section{Summary}

We have observed a number of molecules, in several transitions in the central positions of NGC 4945 and the Circinus galaxy. From a mean-escape probability analysis of the data we conclude the following:

1. In NGC 4945 the CO traces molecular hydrogen densities of $n_{\mathrm{H}_{2}} \approx 310^{3} \mathrm{~cm}^{-3}$ and $\mathrm{HCN}, \mathrm{CS}$ and $\mathrm{HCO}^{+}$ trace densities of $\sim 10^{4} \mathrm{~cm}^{-3}$;

2. In Circinus these values are $\approx 210^{3} \mathrm{~cm}^{-3}$ and $\sim 10^{5} \mathrm{~cm}^{-3}$.

This result implies that the molecular gas in Circinus may be found in denser cores than in NGC 4945. In conjunction with the (relatively) high HCN/CO (and possibly HCN/FIR and radio/FIR) ratio(s) in NGC 4945, this result suggests that much of the HCN and FIR radiation may arise from the AGN rather than the star-burst ${ }^{27}$ (up to $50 \%$ of the bolometric luminosity in NGC 4945 may be due to the AGN, Spoon et al. 2000). If this is to be believed, then previous calculations of the star formation rate in this galaxy may be significant overestimates.

We also find that the kinetic temperature of the gas to be $T_{\text {kin }}=100 \mathrm{~K}$ and $T_{\text {kin }}=50-80 \mathrm{~K}$ in NGC 4945 and Circinus, respectively (from $\mathrm{H}_{2}$ lines, Spoon et al. 2000 also find warm gas, $T_{\mathrm{ex}}=160 \mathrm{~K}$, in NGC 4945). Could the higher temperature in NGC 4945 be related to the asymmetric shapes of (the temperature tracing) molecular profiles? A temperature gradient across the observed

${ }^{24}$ Regarding the previous discussion, although the ratio in NGC 253, is similar to that in M 82 and NGC 4945, perhaps indicating similar conditions, Takano et al. (1995), from the ratios of various other molecular species, postulate additional processes to the formation of molecules in NGC 253, compared to the rest of their sample.

${ }^{25}$ Ratios $\leq 1$ are found in conjunction with outflows, in which $\mathrm{SO}$ is enhanced in molecular shocks (e.g. Pineau des Forêts et al. 1993).

${ }^{26}$ Also values of $\approx 1$ for the column density ratio are found in molecular clouds in the Magellanic clouds (Heikkilä 1998).

27 The HCN being located in the obscuration (dense component of the molecular ring) surrounding the AGN (Shlosman et al. 1990; Friedli \& Martinet 1993; Antonucci 1993; Shaw et al. 1993; Wilson \& Tsvetanov 1994; Maiolino \& Rieke 1995; Fosbury et al. 1999; Conway 1999; Kohno et al. 1999; Curran et al. 2000). It is possible that some of the higher density $\left(10^{5} \mathrm{~cm}^{-3}\right)$ gas in Circinus is also located close to the obscuration but this would be confined to within a few pc (Matt et al. 1996; Curran 2000), and so would not contribute significantly to the CS luminosity due to a low beam filling factor. 
region could cause the possible self-absorption: Molecular rings (which have been insinuated in both these galaxies) are expected to have higher temperatures in their inner radius due to heating from the AGN and star formation, and so we may expect such asymmetric profiles in both cases. The fact that this is not seen in the Circinus spectra may simply imply a smaller gradient in accordance with the lower temperature derived. We hope that future interferometric (ALMA) studies of these galaxies will answer this through detailed mapping of temperature tracing molecules.

We find column densities (per unit line-width) of $\approx 210^{17} \mathrm{~cm}^{-2}\left(\mathrm{~km} \mathrm{~s}^{-1}\right)^{-1}$ for ${ }^{12} \mathrm{CO}$ in both galaxies. Combined with the ${ }^{13} \mathrm{CO}$ results, these values suggest a ${ }^{12} \mathrm{C} /{ }^{13} \mathrm{C}$ ratio of $\approx 50$ for $\mathrm{NGC} 4945$ and $\approx 60-80$ for Circinus. These values are typical of Galactic clouds $(\approx 40-90$, Goldsmith 1987), but higher than in the Galactic centre.

We also report the first detection of $\mathrm{SO}$ in these galaxies, thus increasing the number of extragalactic SO detections to six (Petuchowski \& Bennett 1992; Johansson et al. 1994; Takano et al. 1995; Chin et al. 1998; Heikkilä et al. 1999).

Finally, worth noting is the fact that both the MEP and LTE methods suggest that the gas in NGC 4945 is warmer than in Circinus (by roughly the same factor), and so perhaps LTE methods could provide a useful simple means of determining the relative gas temperatures in a larger sample of Seyfert galaxies.

Acknowledgements. We wish to thank the referee Christian Henkel for his helpful comments, Francisco Azagra at SEST for locating some of the older data for us and the operators in general for their help with the observations. Thanks also to Michael Olberg, John Black and Silvana Nikolić at Onsala for their help with RADEX. Also at Onsala, Antonis Polatidis who made modifications (not possible from SEST) to Figs. 3 and 4. AH acknowledges financial support from the Academy of Finland through grant No. 1011055.

\section{References}

Aalto, S., Black, J. H., Booth, R. S., \& Johansson, L. E. B. 1991, A\&A, 247, 291

Ables, J. G., Foster, J. R., Manchester, R. N., et al. 1987, MNRAS, 226, 157

Antonucci, R. R. J. 1993, ARA\&A, 31, 473

Batchelor, R. A., Jauncey, D. L., \& Whiteoak, J. B. 1982, MNRAS, 200, 19

Bergin, E. A., \& Langer, W. D. 1997, ApJ, 486, 316

Bergman, P., Aalto, S., Black, J. H., \& Rydbeck, G. 1992, A\&A, 265, 403

Cecil, G., Wilson, A. S., \& Tully, R. B. 1992, ApJ, 390, 365

Chin, Y. N., Henkel, C., Millar, T. J., Whiteoak, J. B., \& Marx-Zimmer, M. 1998, A\&A, 330, 901

Christopoulou, P. E., Holloway, A. J., Steffen, W., et al. 1997, MNRAS, 284, 385

Conway, J. 1999, HI Absorption from a Circumnuclear TORUS in the Hidden Quaser Cygnus A, in Highly Redshifted Radio Lines, ed. C. Carilli, S. Radford, K. Menton, \& G. Langston, ASP Conf. Ser., 259
Curran, S. J., Aalto, S., \& Booth, R. S. 2000, A\&AS, 141, 193

Curran, S. J., Johansson, L. E. B., Rydbeck, G., \& Booth, R. S. 1998, A\&A, 338, 863

Curran, S. J. 2000, Ph.D. Thesis, Chalmers University of Technology

Dahlem, M., Golla, G., Whiteoak, J. B., et al. 1993, A\&A, 270, 29

de Grijp, M. H. K., Miley, G. K., Lub, J., \& de Jong, T. 1985, Nature, 314, 240

de Vaucouleurs, G., Peters, W. L., Bottinelli, L., Gougenheim, L., \& Paturel, G. 1981, ApJ, 248, 408

Dickens, J. E., \& Irvine, W. M. 1999, ApJ, 518, 733

dos Santos, P. M., \& Lépine, J. R. D. 1979, Nature, 278, 34

Elmouttie, M., Krause, M., Haynes, R. F., \& Jones, K. L. 1998, MNRAS, 300, 1119

Elmouttie, M., Haynes, R. F., \& Jones, K. L. 1997, Proc. Astron. Soc. Aust., 14, 140

Forbes, D. A., \& Norris, R. P. 1998, MNRAS, 300, 757

Fosbury, R. A. E., Vernet, J., Villar-Martin, M., et al. 1999, Optical Continuum Structure of Cygnus A, in KNAW colloqium on: The Most Distant Radio Galaxies, ed. P. Best, \& M. Lehnert (Reidel, Amsterdam), 311

Freeman, K. C., Karlsson, B., Lyngå, G., et al. 1977, A\&A, 55, 445

Friedli, D., \& Martinet, L. 1993, A\&A, 277, 27

Gardner, F. F., \& Whiteoak, J. B. 1982, MNRAS, 201, 13p

Goldsmith, P. F. 1987, Molecular clouds: An overview, in Interstellar Processes, ed. D. J. Hollenbach, \& H. A. Thronson (Reidel, Dordrecht), 51

Greenhill, L. J., Jiang, D. R., Moran, J. M., et al. 1995, ApJ, 440,619

Greenhill, L. J., Ellingsen, S. P., Norris, R. P., et al. 1997, ApJ, 474, L103

Greenhill, L. J., Moran, J. M., \& Herrnstein, J. R. 1997, ApJ, 481, L23

Greenhill, L. J. 2001, Accretion and Outflow in the Circinus AGN, in Proceedings of the 5th EVN Symposium, ed. J. Conway, A. Polatidis, \& R. Booth, Chalmers University of Technology, Göteborg, Sweden, 101

Gu, Q. S., Huang, J. H., Su, H., \& Shang, Z. H. 1997, A\&A, 319, 92

Gu, Q. S., Huang, J. H., \& Ji, L. 1999, Astrophys. Space Sci., 260,389

Harnett, J. I., Whiteoak, J. B., Reynolds, J. E., Gardner, F. F., \& Tzioumis, A. 1990, MNRAS, 244, 130

Harrison, A., Puxley, P., Russell, A., \& Brand, P. 1998 MNRAS, 297, 624

Heikkilä, A., Johansson, L. E. B., \& Olofsson, H. 1999, A\&A, 344,817

Heikkilä, A. 1998, Ph.D. Thesis, Chalmers University of Technology

Helfer, T. T., \& Blitz, L. 1993, BAAS, 183, 3007

Helfer, T. T., \& Blitz, L. 1995, ApJ, 450, 90

Henkel, C., Whiteoak, J. B., Nyman, L.-Å., \& Harju, J. 1990, A\&A, 230, L5

Henkel, C., Whiteoak, J. B., \& Mauersberger, R. 1994, A\&A, 284, 17

Ho, L. C., Filippenko, A. V., Sargent, W. L. W., \& Peng, C. Y. 1997, ApJS, 112, 391

Hummel, E., van Gorkom, J. H., \& Kotanyi, C. G. 1983, ApJ, 267, L5

Hüttemeister, S., Henkel, C., Mauersberger, R., et al. 1995, A\&A, 295, 571 
Israel, F. P. 1992, A\&A, 265, 487

Jackson, J. M., Paglione, T. A. D., Carlstrom, J. E., \& Nguyen-Q-Rieu, 1995, ApJ, 438, 695

Jansen, D. 1995, Ph.D. Thesis, Leiden, The Netherlands

Johansson, L. E. B., Aalto, S., Booth, R. S., \& Rydbeck, G. 1991, Molecular Gas in the Circinus Galaxy, in Dynamics of Disc Galaxies, ed. B. Sundelius, University of Göteborg and Chalmers University of Technology, Göteborg, 249

Johansson, L. E. B., Olofsson, H., Hjalmarson, Å., Gredel, R., \& Black, J. H. 1994, A\&A, 291, 89

Jones, K. L., Koribalski, B. S., Elmouttie, M., \& Haynes, R. F. 1999, MNRAS, 302, 649

Kohno, K., Kawabe, R., Vila-Vilaró, B. 1999, NMA Survey of $\mathrm{CO}$ and HCN Emission from Nearby Active Galaxies, in Proceedings of the 3rd Cologne-Zermatt Symposium, The Physics and Chemistry of the Interstellar Medium, ed. V. Ossenkopf, J. Stutzki, \& G. Winnewisser, 2251

Koornneef, J. 1993, ApJ, 403, 581

Lee, H.-H., Bettens, R. P. A., \& Herbst, E. 1996, A\&AS, 119, 111

Leung, C., \& Liszt, H. S. 1976, ApJ, 208, 732

Lonsdale, C. J., Helou, G., Good, J. C., \& Rice, W. 1985, Cataloged Galaxies and Quasars Observed in the IRAS Survey, Jet Propulsion Laboratory, Pasadena

Maiolino, R., \& Rieke, G. H. 1995, ApJ, 454, 95

Marconi, A., Moorwood, A. F. M., Origlia, L., \& Oliva, E. 1994, ESO Messenger, 78, 20

Matt, G., Fiore, F., Perola, G. C., et al. 1996, MNRAS, 281, L69

Mauersberger, R., Henkel, C., Whiteoak, J. B., Chin, Y. N., \& Tieftrunk, A. R. 1996, A\&A, 309, 705

Moorwood, A. F. M., \& Glass, I. S. 1984, A\&A, 135, 281

Moorwood, A. F. M., \& Oliva, E. 1994, ApJ, 429, 602

Moorwood, A. F. M., Lutz, D., Oliva, E., et al. 1996a, A\&A, 315, L109

Moorwood, A. F. M., Van Der Werf, P. P., Kotilainen, J. K., Marconi, A., \& Oliva, E. 1996b, A\&A, 308, L1

Nakai, N., Hayashi, M., Handa, T., et al. 1987, PASJ, 39, 685

Nakai, N., Inoue, M., Miyazawa, K., Miyoshi, M., \& Hall, P. 1995, PASJ, 47, 771

Nilsson, A., Hjalmarson, Å., Bergman, P., \& Millar, T. 2000, A\&A, 358, 257

Oliva, E., Salvati, M., Moorwood, A. F. M., \& Marconi, A. 1994, A\&A, 288, 457

Oliva, E., Marconi, A., Cimatti, A., \& Alighieri, S. D. S. 1998, A\&A, 329, L21

Osterbrock, D. E. 1989, Astrophysics of Gaseous Nebulae and Active Galactic Nuclei (University Science Books, Mill Valley, California)

Papadopoulos, P. P., \& Seaquist, E. R. 1998, ApJ, 492, 521

Pedlar, A., Meaburn, J., Axon, D. J., et al. 1989, MNRAS, 238,863
Petuchowski, S. J., \& Bennett, C. L. 1992, ApJ, 391, 137

Pineau des Forêts, G., Roueff, E., Schilke, P., \& Flower, D. R. 1993, MNRAS, 262, 915

Prantzos, N., Aubert, O., \& Audouze, J. 1996, A\&A, 309, 760

Radovich, M., Rafanelli, P., \& Barbon, R. 1998, A\&A, 334, 124

Rice, W., Lonsdale, C. J., Soifer, B. T., et al. 1988, ApJS, 68, 91

Rigopoulou, D., Papadakis, I., Lawrence, A., \& Ward, M. 1997, A\&A, 327, 493

Roy, A. L., Norris, R. P., Kesteven, M. J., Troup, E. R., \& Reynolds, J. E. 1998, MNRAS, 301, 1019

Sage, L. J., Shore, S. N., \& Solomon, P. M. 1990, ApJ, 351, 422

Shaw, M. A., Combes, F., Axon, D. J., \& Wright, G. S. 1993, A\&A, 273, 31

Shlosman, I., Begelman, M. C., \& Frank, J. 1990, Nature, 345, 679

Solomon, P. M., Downes, D., \& Radford, S. J. E. 1992, ApJ, 387, L55

Solomon, P. M., Radford, S. J. E., \& Downes, D. 1990, ApJ, 348, L53

Spoon, H. W. W., Koornneef, J., Moorwood, A. F. M., Lutz, D., \& Tielens, A. G. G. M. 2000, A\&A, 357, 898

Sternberg, A., Genzel, R., \& Tacconi, L. 1994, ApJ, 436, L131

Storchi-Bergmann, T., Winge, C., Ward, M. J., \& Wilson, A. S. 1999, MNRAS, 304, 35

Storchi-Bergmann, T., Kinney, A. L., \& Challis, P. 1995, ApJS, 98, 103

Tacconi, L. J., Genzel, R., Blietz, M., et al. 1994, ApJ, 426, L77

Tacconi, L., Schinnerer, E., Eckart, A., et al. 1998, Astronomische Gesellschaft Meeting Abstracts, Abstracts of Contributed Talks and Posters presented at the Annual Scientific Meeting of the Astronomische Gesellschaft at Heidelberg, September 14-19, 1998, talk \#F01, 14, 01

Takano, S., Nakai, N., \& Kawaguchi, K. 1995, PASJ, 47, 801

Turner, B. E. 1991, ApJS, 76, 617

Ulvestad, J. S., \& Antonucci, R. R. J. 1997, ApJ, 488, 621

Vogler, A., \& Pietsch, W. 1999, A\&A, 342

Whiteoak, J. B., \& Gardner, F. F. 1975, ApJ, 195, L81

Whiteoak, J. B., \& Gardner, F. F. 1985, Proc. Astron. Soc. Aust., 6, 171

Whiteoak, J. B., \& Wilson, W. E. 1990, ApJ, 245, 665

Whiteoak, J. B., Dahlem, M., Wielebinski, R., \& Harnett, J. I. 1990, A\&A, 231, 25

Whittle, M., Pedlar, A., Meurs, E. J. A., et al. 1988, ApJ, 326, 125

Whittle, M. 1992, ApJ, 387, 121

Wills, K. A., Pedlar, A., Muxlow, T. W. B., \& Stevens, I. R. 1999, MNRAS, 305, 680

Wilson, A. S., \& Tsvetanov, Z. I. 1994, AJ, 107, 1227

Young, J. S., \& Scoville, N. 1982, ApJ, 258, 467 